\title{
Integration of electro-active $\pi$-conjugated units in nanosegregated liquid-crystalline phases
}

\begin{abstract}
Masahiro Funahashi
Unlike conventional liquid crystals bearing alkyl chains, perylene tetracarboxylic bisimide (PTCBI) derivatives bearing oligosiloxane moieties were synthesized. The oligosiloxane moieties are bulky and have a tendency to form a liquid-like conformation due to the low rotation potential of $\mathrm{Si}-\mathrm{O}$ bonds. The PTCBI derivatives bearing oligosiloxane chains exhibit the columnar phase at room temperature. They are soluble in various organic solvents, and thin films can be produced by a spin-coating method. In the columnar phase, crystal-like $\pi$-stacks are surrounded by a liquid-like mantle consisting of the oligosiloxane moieties of the PTCBI derivatives, resulting in efficient electron transport. Even the PTCBI derivatives bearing polymerizable cyclotetrasiloxane rings show the liquid-crystalline (LC) phases at room temperature. The spin-coated films of the LC PTCBI derivatives with cyclotetrasiloxane rings can be polymerized and insolubilized by exposure to the vapors of trifluoromethanesulfonic acid. PTCBI derivatives bearing oligosiloxane moieties and a triethylene oxide chain coordinated to ionic species exhibit a dimeric lamellar phase in which ion-conductive sublayers and electron-transporting $\pi$-stacks are separately self-organized. Phenylterthiophene derivatives bearing oligosiloxane moieties show a ferroelectric smectic $\mathrm{C}^{*}$ phase in which the anomalous photovoltaic effect is observed.
\end{abstract}

Polymer Journal (2017) 49, 75-83; doi:10.1038/pj.2016.91; published online 5 October 2016

\section{INTRODUCTION}

Control of the self-organization of electro- and redox-active $\pi$ conjugated units is indispensable to the design of organic functional materials for electronic and optic applications. In particular, the integration of these functional moieties in soft matrixes such as polymers has great potential for use in flexible, lightweight devices. ${ }^{1}$

The self-organization of $\pi$-conjugated units in the liquid crystal phase enhances carrier transport properties. Liquid-crystalline (LC) semiconductors, including columnar, smectic and nematic compounds, have been studied by European and Japanese researchers. ${ }^{2-5}$ In the LC phases, electronic charge carriers are transported by the hopping mechanism, although band-like conduction has been reported in ordered smectic phases. ${ }^{6,7}$ In addition to basic research, the application of these materials to electronic devices, such as light emitting diodes, ${ }^{8}$ field-effect transistors ${ }^{9}$ and solar cells, ${ }^{10,11}$ has been studied. The self-organization of $\pi$-conjugated units also affects the electronic properties of organo-gels ${ }^{12-15}$ and conductive polymers. ${ }^{16-18}$

Unlike organic crystals and amorphous solids, supramolecular structures can be constructed in liquid crystal phases using nanosegregation between immiscible moieties built into LC molecules. ${ }^{19}$ Kato and coworkers reported one- and two-dimensional ionic conduction in nanosegregated supramolecular liquid crystals. ${ }^{20}$ Rod-like LC molecules bearing polar oligoethylene oxide chains form two-dimensional LC superstructures in which a liquid-like, ion-conductive mantle of oligoethylene oxide chains are separated by inactive sublayers consisting of rigid core moieties. Gallic acid derivatives bearing long alkyl chains and an imidazolium moiety assemble into one-dimensional columnar aggregates in the LC phases, resulting in one-dimensional ionic transport. ${ }^{21}$

For LC electro-active materials, LC $\pi$-conjugated molecules bearing immiscible moieties possess the remarkable feature in which nanostructures can be constructed by nanosegregation. ${ }^{22}$ Polycatenar oligothiophene derivatives demonstrate nanosegregated smectic, columnar, micellar cubic phases depending upon the volume balance between the aromatic and alkyl units. ${ }^{23}$ Oligoithiophene bearing lateral hydrophilic substituents forms various supramolecular LC structures. ${ }^{24}$ Figure 1 displays an example of the functionalization of $\pi$-conjugated LC materials based on nanosegregation. Phenylterthiophene derivatives bearing an imidazolium unit exhibit nanosegregated smectic phases in which hole-transporting redox-active layers and ion-conductive layers are integrated separately. ${ }^{25,26}$ As shown in Figure 1, the DC bias application induces the formation of an electrical double layer that promotes hole injection from the anode to the redox active layers. This coupling of the electronic function of the $\pi$-conjugated units with the ionic conductivity causes anodic oxidation, resulting in electrochromism without an electrolyte solution. Nanosegregated LC structures can integrate molecular functions, resulting in multifunctional materials in which electronic properties cooperate with other characteristics. In this article, the recent results on the electronic functions of $\pi$-conjugated LC materials based on nanosegregated dynamic structures are reviewed. 


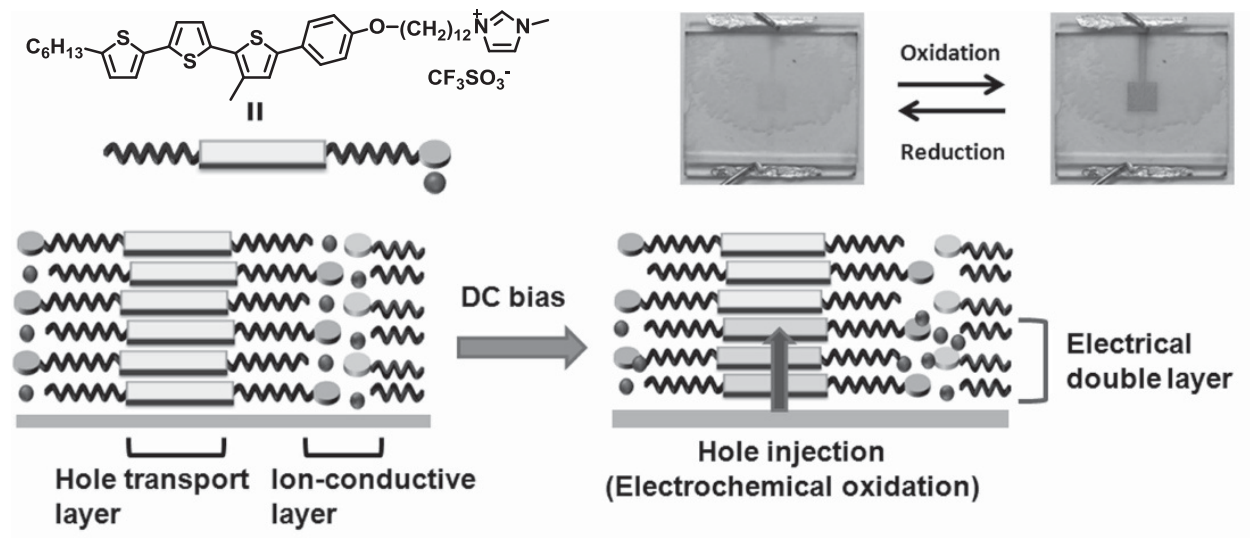

Figure 1 Schematic illustration of electrochromism in the nanosegregated smectic phase of a phenylterthiophene derivative bearing an imidazolium moiety.

\section{NANOSEGREGATED COLUMNAR PHASES OF PERYLENE BISIMIDE DERIVATIVES BEARING OLIGOSILOXANE CHAINS}

In general, organic compounds comprised of extended $\pi$-conjugated systems exhibit high crystallinity that results in low solubility in organic solvents. This property makes it difficult to purify the materials by column chromatography and recrystallization. The melting points of these materials are sometimes higher than their decomposition temperatures, and the thin film preparations are difficult both by vacuum and solution processes. For liquid crystal materials, we sometimes encounter difficulties in capillaryfilling of the sample into a liquid crystal cell or in the production of thin films using a spin-coating method. ${ }^{27}$

In the field of organic electronics, the introduction of alkyl chains to extended $\pi$-conjugated units is recognized as an effective method to improve the processability of organic semiconductors, including molecular crystals and conjugated polymers. Although alkyl chains increase the solubility of the organic semiconductor molecules due to their thermal motion, ${ }^{27}$ they also promote self-organization of the organic molecules. This tendency is remarkable in $\pi$-conjugated polymers. ${ }^{28}$ However, the introduction of alkyl chains is not sufficient to increase the solubilities of organic semiconductors based on large $\pi$-conjugated moieties, which are favorable for efficient charge carrier transport and visible light absorption. The introduction of excessive alkyl chains or bulky moieties often inhibit supramolecular packing of $\pi$-conjugated systems, resulting in low carrier mobility.

Therefore, we have given higher consideration to oligosiloxane chains. Cage-shaped silsesquoxane has been used as a integrating unit in dendric LC oligomers. ${ }^{29}$ Linear oligosiloxane chains, such as 1,1,1,3,3,5,5-heptamethyl-2,4-trisiloxane and 1,1,1,3,3-pentamethyl-2disiloxane, were introduced to the terminals of the alkyl side chains of rod-like LC molecules. ${ }^{30}$ Compared with alkyl chains, which have a tendency to form an all-trans conformation, oligosiloxane chains consist of $\mathrm{Si}-\mathrm{O}$ bonds with longer bond lengths than $\mathrm{C}-\mathrm{C}$ bonds and low rotational potentials, resulting in their remarkable thermal motion. However, layer structures of smectic phases are thermodynamically stabilized by the effects of oligosiloxane chain nanosegregation. ${ }^{30}$ In de Vries type ferroelectric liquid crystals, a temperature-independent layer spacing of the ferroelectric LC phase has been reported. ${ }^{31}$ Only one example of columnar liquid crystals of triphenylene derivatives bearing six oligosiloxane chains has been reported. ${ }^{32}$ For side chain polymers, high hole mobility was observed in a nanosegregated smectic phase in which rigid side chain mesogenic terthiophene units and liquid-like polysiloxane chains were microscopically separated. ${ }^{33}$
Perylene tetracarboxylic bisimide (PTCBI) derivatives are typical n-type organic semiconductors. ${ }^{34}$ To increase the solubility and control the aggregation states of the $\pi$-conjugated units, various alkylated derivatives have been synthesized. PTCBI derivatives bearing branched alkyl chains were reported by Langhals and coworkers. ${ }^{35}$ Würthner and coworkers synthesized LC PTCBI derivatives bearing 4 or 6 alkyl chains. ${ }^{36}$ Supramolecular aggregations of PTCBI derivatives based on hydrogen-bonding have also been reported. ${ }^{37}$ In this study, we synthesized LC PTCBI derivatives bearing oligosiloxane moieties at the terminal positions of the alkyl side chains.

The LC PTCBI derivatives bearing four oligosiloxane moieties were synthesized by the hydrosilylation reaction between PTCBI derivatives bearing alkenyl side chains and hydrooligosiloxane in the presence of Karstedt's catalyst. Column chromatography and repeated re-precipitation gave sufficiently pure samples in good yields. ${ }^{38,39}$

These LC PTCBI derivatives bearing oligosiloxane chains exhibit columnar phases at room temperature. The phase transition properties are summarized in Figure 2. Although PTCBI derivative 1 bearing trisiloxane chains exhibits a hexagonal columnar disordered phase, compounds 2 and 3 bearing disiloxane chains exhibit the hexagonal and rectangular ordered columnar phases. ${ }^{38,39}$ Compound 4, which bears longer alkyl spacers, exhibits a disordered columnar phase. The most prominent feature of these columnar phases is the interdigitation of the oligosiloxane chains. Figure 3a displays the X-ray diffraction patterns of the hexagonal columnar phases of compounds 1 and 2. In the hexagonal phases, the lattice constants were 24.8 for compound $\mathbf{1}$ and 22.2 for compound 2 . These values are shorter than the molecular lengths ( 36 and $32 \AA$ for compounds 1 and 2, respectively). Figure $3 \mathrm{~b}$ shows a schematic illustration of the hexagonal columnar phase of compound 2. These results indicate that the oligosiloxane chains interdigitate to stabilize the columnar aggregates due to the attractive interaction between the oligosiloxane moieties, as shown in Figure 3b. In other words, nanosegregation between rigid aromatic cores and liquid-like oligosiloxane moieties should be a driving force of the formation of the columnar aggregates. ${ }^{39}$

These compounds are soluble in organic solvents except for alcohols. Notably, they are soluble in non-polar $n$-hexane and cyclohexane. The solubility of compound 2 exceeds $30 \mathrm{wt} \%$ in toluene. LC thin films of these compounds can be produced by a spin-coating method. The thickness of the thin films can be controlled between $1 \mu \mathrm{m}$ and several tens of $\mathrm{nm}$ by varying the concentration and the rotation speed. In the spin-coated thin films, the columnar aggregates align parallel to the surface of the substrate, whereas in sandwich-type samples, the columnar aggregates sometimes stand perpendicular to 

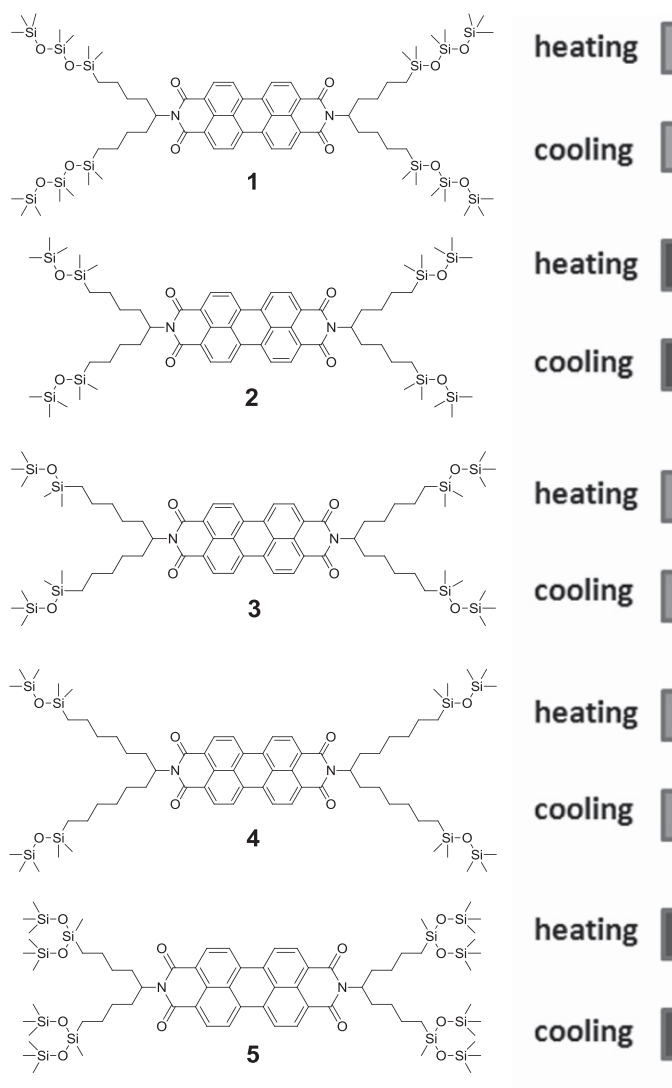

\begin{tabular}{|l|ll|}
\hline Col hd & \multicolumn{1}{c|}{ Iso } \\
\hline \multicolumn{3}{|c|}{$51^{\circ} \mathrm{C}$} \\
\hline Col hd & & Iso \\
\hline
\end{tabular}

heating

\begin{tabular}{|c|c|c|}
\hline $\mathrm{Col}_{\mathrm{ro}}$ & $\mathrm{Col}_{\text {ho }}$ & Iso \\
\hline
\end{tabular}

cooling

\begin{tabular}{l|ll|l|}
$\mathrm{Col}_{\text {ro }}$ & \multicolumn{2}{|c|}{$\mathrm{Col}_{\text {ho }}$} & Iso \\
\hline \multicolumn{3}{c}{$32^{\circ} \mathrm{C}$} & $135^{\circ} \mathrm{C}$
\end{tabular}

heating

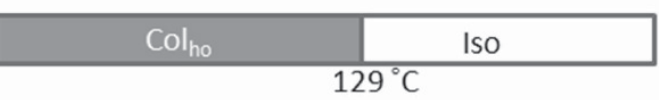

cooling

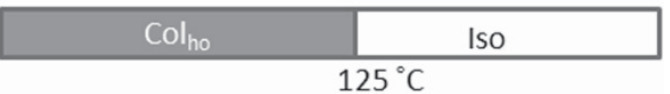

heating

\begin{tabular}{|l|l|}
\hline Col $_{\text {ho }}$ & Iso \\
\hline \multicolumn{3}{|c|}{$112^{\circ} \mathrm{C}$} \\
\hline
\end{tabular}

cooling

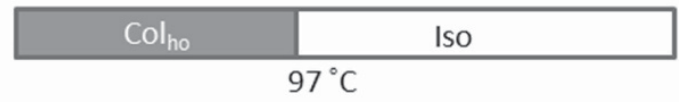

heating

\begin{tabular}{|lll|}
\hline Col $_{\text {ro }}$ & Iso \\
\hline \multicolumn{3}{|c|}{$122^{\circ} \mathrm{C}$} \\
\hline Col $_{\text {ro }}$ & & Iso \\
\hline & $112{ }^{\circ} \mathrm{C}$ & \\
\hline
\end{tabular}

Figure 2 Phase transition behavior of the LC PTCBI derivatives. $\mathrm{Col}_{\text {ho }}$, Col $\mathrm{hd}_{\text {, }}$ and $\mathrm{Col}_{\mathrm{ro}}$ denote columnar hexagonal ordered, columnar hexagonal disordered and columnar rectangular ordered phases, respectively.
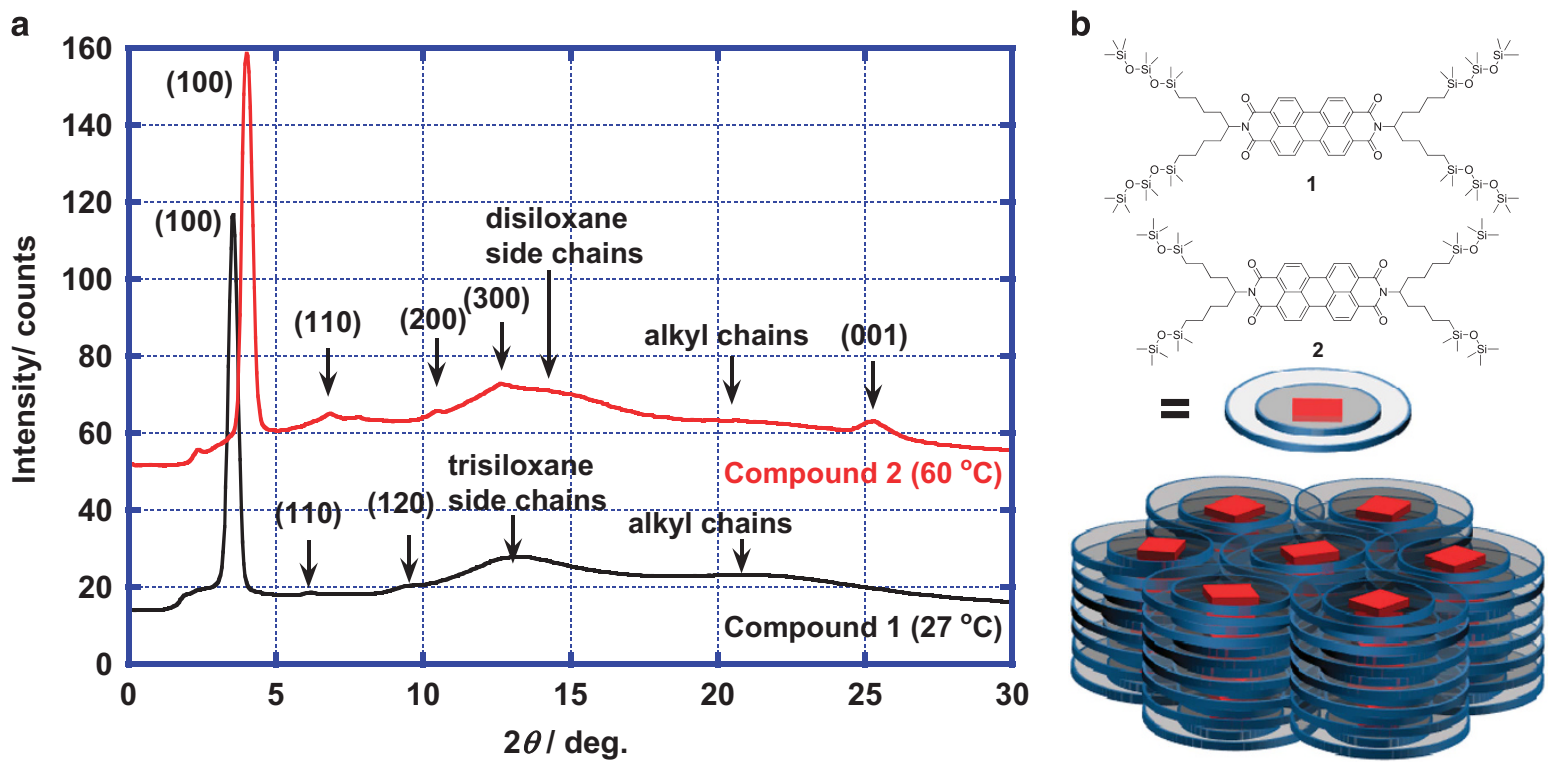

Figure 3 (a) X-ray diffraction pattern of the hexagonal columnar phases of compound $\mathbf{1}$ at $27^{\circ} \mathrm{C}$ and compound 2 at $60^{\circ} \mathrm{C}$. (b) Schematic illustration of the hexagonal columnar phases of compounds 1 and 2 .

the substrate surface. Uniaxially aligned thin films can be produced using the friction transfer method. ${ }^{39}$ The columnar phases of compound 2 are thermodynamically stable, and columnar aggregates in the thin film states do not crystallize.
The electron mobilities in the columnar phases were determined by a time-of-flight method. The electron mobility exceeded $0.1 \mathrm{~cm}^{2} \mathrm{~V}^{-1} \mathrm{~s}^{-1}$ in the rectangular ordered columnar phase of compound 2 at room temperature. ${ }^{39}$ In the rectangular ordered columnar phase, the 
molecular position presents a periodical order within the columnar aggregates, and the intermolecular distance is $3.48 \AA$, which is a typical $\pi-\pi$ stacking distance. These large intermolecular $\pi$-orbital overlaps should contribute to the efficient electron transport in the columnar aggregates in the LC phase of compound 2. The hole and electron mobilities in the ordered columnar phases of hexaalkyloxytriphenylene derivatives are on the order of $10^{-2} \mathrm{~cm}^{2} \mathrm{~V}^{-1} \mathrm{~s}^{-1}$. The electron mobilities in the ordered columnar phases of these LC PTCBI derivatives bearing disiloxane chains are higher than those of the ordered columnar phases of hexaalkoxytriphenylene derivatives. The electron transport mechanism is discussed in section 4 .

It should be noted that the PTCBI derivative $\mathbf{5}$ bearing branched trisiloxane moieties also exhibits the rectangular columnar ordered phase. ${ }^{40}$ In the presence of the bulky, branched trisiloxane units, a periodical order of the molecular position within the columnar aggregates based on $\pi-\pi$ stacking was observed in the ordered columnar phase of compound 5. In terms of the closed molecular packing within the columnar aggregates, the electron mobility exceeded $0.1 \mathrm{~cm}^{2} \mathrm{~V}^{-1} \mathrm{~s}^{-1}$ at the elevated temperature.

\section{IN SITU POLYMERIZATION OF PTCBI DERIVATIVES BEARING CYCLOTETRASILOXANE RINGS}

Side chains of conventional LC molecules, including rod-like and disk-like mesogens, have mainly consisted of linear alkyl groups, although branched alkyl chains are sometimes used. An exception of liquid crystals bearing crown ether moieties as either core or side chain components have been reported. ${ }^{41,42}$

The classical description of the role of side chains in LC molecules is that their thermal motion moderately weakens the strong interactions between rigid core moieties to induce mesophases. Considering the aspect of molecular motion, Ohta and coworkers designed 'flying seed' LC molecules to verify that mesophases can be induced by the thermal motion of bulky units other than alkyl chains. ${ }^{43}$ We have given consideration to another aspect of the role of the side chains.
As mentioned in the former section, nanosegregation is a main driving force of the formation of columnar LC phases of the PTCBI derivatives bearing oligosiloxane moieties. ${ }^{39}$ This nanosegregation should be caused not only by linear oligosiloxane chains.

We synthesized PTCBI derivatives bearing cyclotetrasiloxane rings, which are bulkier than linear oligosiloxane chains. By the hydrosilylation reaction between commercially available 1,1,3,3,5,5,7-heptamethyl-2,4,6,8-cyclotetrasiloxane and PTCBI derivatives bearing alkenyl chains, compounds $\mathbf{6 - 8}$ were obtained in good yields. ${ }^{44,45}$

The phase transition behaviors of PTCBI derivatives 6-8 are summarized in Figure 4. Compound 7 exhibits monotropic columnar phases and the LC phases crystallizes immediately. In contrast, compound 8 exhibits a metastable hexagonal columnar phase that changes to a thermodynamically stable rectangular columnar phase at room temperature in a couple of months. In these columnar phases, the periodical order of the molecular position does not exist within the columnar aggregates, and these LC phases are assigned to hexagonal and rectangular columnar disordered phases. The bulky cyclotetrasiloxane rings should inhibit close packing of $\pi$-conjugated cores. However, the electron mobility exceeds $0.1 \mathrm{~cm}^{2} \mathrm{~V}^{-1} \mathrm{~s}^{-1}$ in the disordered columnar phase of compound $\mathbf{8}$ at room temperature. Compound $\mathbf{8}$ is also soluble in various organic solvents, and thin films can be prepared by the spin-coating method. These deposited thin films have thicknesses of several hundred $\mathrm{nm}$ and are in the hexagonal columnar ordered phase, which is stable over several months.

Next, the in situ polymerization of the LC phase of compound $\mathbf{8}$ was investigated. Cyclotetrasiloxane moieties polymerized in the presence of an acidic or basic catalyst through the ring-opening mechanism. Compound $\mathbf{8}$ has four reactive sites, and due to the action of an acid or base, it forms only insoluble precipitates in the solution state. In the hexagonal columnar phase of compound $\mathbf{8}$, the lattice constant is much shorter than the extended molecular length, indicating interdigitation between the cyclotetrasiloxane rings.
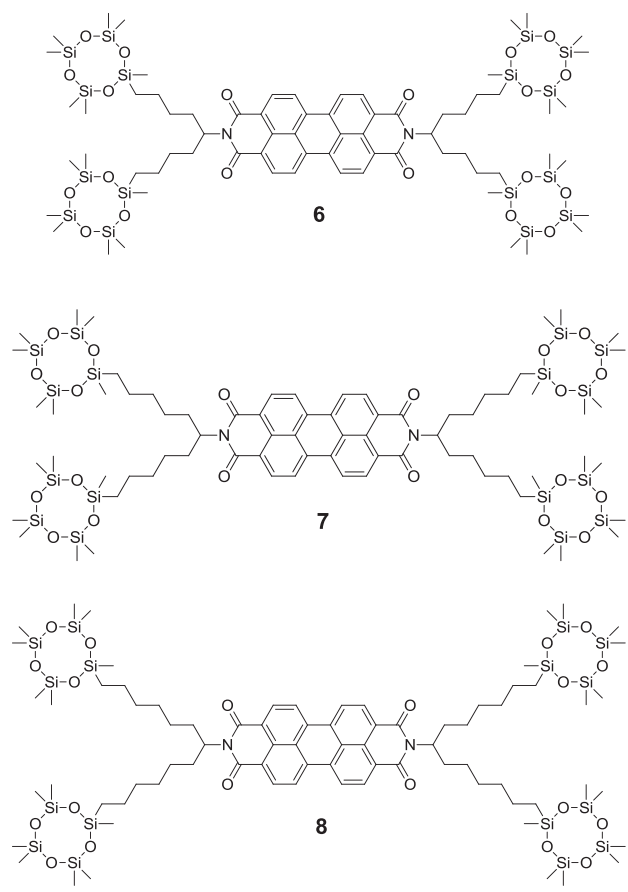

heating

\begin{tabular}{|c|c|}
\hline Cryst & Iso \\
\hline
\end{tabular}

cooling

\begin{tabular}{|c|c|}
\hline Cryst & Iso \\
\hline & $71{ }^{\circ} \mathrm{C}$ \\
\hline
\end{tabular}

heating

\begin{tabular}{|c|c|}
\hline Cryst & Iso \\
\hline \multicolumn{2}{|c|}{$103^{\circ} \mathrm{C}$} \\
\hline Col $\rightarrow$ Cryst & Iso \\
\hline
\end{tabular}

cooling

$68^{\circ} \mathrm{C}$

heating

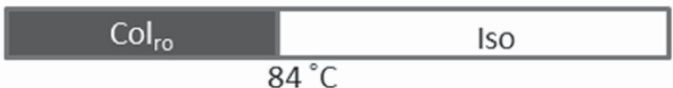

cooling

$\mathrm{Col}_{\text {ho }} \rightarrow \mathrm{Col}_{\mathrm{ro}} \longrightarrow$ Iso

Figure 4 Phase transition behaviors of LC PTCBI derivatives bearing cyclotetrasiloxane rings. 
Due to the nanosegregation between the $\pi$-conjugated cores and side chains, the electron-conductive, one-dimensional $\pi$-stacks are surrounded by a polymerizable mantle in the columnar phase. This nanosegregated structure indicates that efficient ring-opening polymerization is possible in the LC thin film state when an acidic or basic catalyst is properly introduced into the thin films. ${ }^{45}$

We found that the exposure of the LC thin film to the vapors of trifluoromethanesulfonic acid is effective for the in situ polymerization of the LC thin films of compound 8, as shown in Figure 5. By spin-coating a toluene solution of compound $\mathbf{8}$ onto glass substrates, LC thin films with a thickness of $200 \mathrm{~nm}$ were produced. A small glass tube containing a few drops of trifluoromethanesufonic acid was placed in a Petri dish and covered with another dish. The dish was left in an oven at $70{ }^{\circ} \mathrm{C}$ for $30 \mathrm{~min}$, during which time the volume of the Petri dish was saturated with the vapors of the acid. A spin-coated thin film was placed in the dish, which was capped with another dish. The capped dish was placed in the oven at $70^{\circ} \mathrm{C}$ for $30 \mathrm{~min}$, during which time the spin-coated thin film was insolubilized by the acid vapor-induced ring-opening polymerization. ${ }^{45}$

As shown in Figure 5, the optical textures of the thin films did not change during the polymerization process, indicating the retention of the columnar structures. The X-ray diffraction patterns, IR transmission spectra and surface morphology observation by atomic force microscopy indicate the presence of columnar structures in the polymerized thin films of compound $\mathbf{8}$.

When non-treated substrates are used, the columnar aggregates align parallel to the surfaces of the thin films. Uniaxially aligned thin films can be produced by the friction transfer method. The polarized absorption spectrum of the uniaxially aligned thin film reveals a dichroic ratio larger than 5:1 before polymerization. After the polymerization, the dichroic ratio decreases to $1: 3$, indicating an increase in the structural disorder. However, the optical anisotropy is retained during the polymerization. ${ }^{45}$

\section{ELECTRON TRANSPORT CHARACTERISTICS IN NANOSEGREGATED COLUMNAR PHASES OF LC PTCBI DERIVATIVES}

The time-of-flight measurement reveals the hopping nature of the electron transport process in the nanosegregated columnar phases of compounds 1 and $2 .{ }^{46}$ The charge carrier mobility determined by the time-of-flight method is a drift mobility affected by disorder and defects. ${ }^{47}$ The measurement of the carrier mobility over wide ranges of temperatures and fields can provide information on the carrier transport mechanism in organic semiconductors. Conventional LC semiconductors exhibit LC phases within narrow, elevated temperature ranges. The carrier transport characteristics of the columnar ordered phases of LC triphenylene dimers, ${ }^{48}$ terthiophene ${ }^{6}$ and phenylterthiophene ${ }^{7}$ derivatives were studied over wide temperature ranges. In contrast to alkyl-substituted LC semiconductors with rigid structures, the electron transport processes in the nanosegregated columnar phases of oligosiloxane-substituted PTCBI derivatives are affected by the dynamic softness of the columnar aggregates.

Figures $6 \mathrm{a}$ and $\mathrm{b}$ exhibits transient photocurrent curves for electrons in the disordered and ordered columnar phases of compounds 1 and 2 at room temperature. In the disordered columnar phase of compound 1, transient photocurrents are slightly dispersive and the electron mobility, which is temperature- and field-dependent, is $\sim 1 \times 10^{-4}$ $\mathrm{cm}^{2} \mathrm{~V}^{-1} \mathrm{~s}^{-1}$. In contrast, completely non-dispersive transient photocurrent curves are observed for electrons in the ordered columnar phase of compound 2, and the electron mobility at room temperature exceeds $0.1 \mathrm{~cm}^{2} \mathrm{~V}^{-1} \mathrm{~s}^{-1}$. At approximately room temperature, the electron mobility is independent of the electric field and the temperature.

Figure $6 \mathrm{c}$ demonstrates the electron mobility of compounds $\mathbf{1}$ and 2 as a function of temperature. The electron mobility in the disordered columnar phase of compound $\mathbf{1}$ decreases with decreasing temperature, indicating the hopping transport of electrons. For the ordered columnar phase of compound 2 , the temperature range is

a

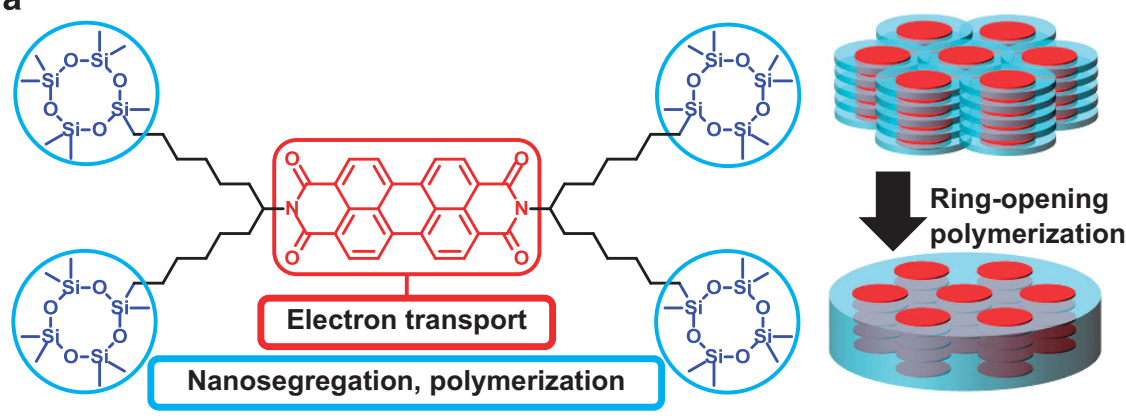

b

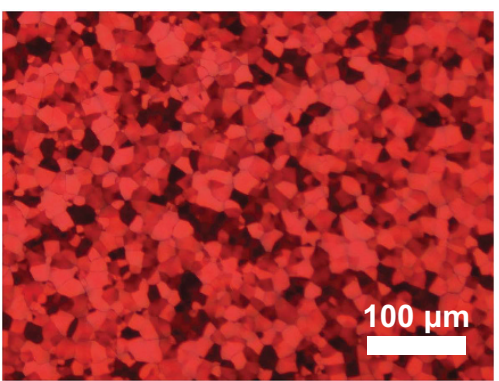

C

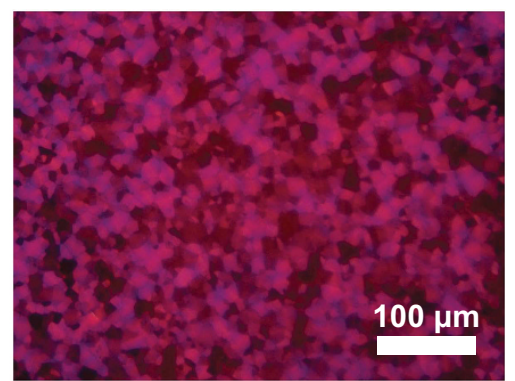

Figure 5 (a) Schematic illustration of the acid-vapor-induced in situ ring-opening polymerization of compound $\mathbf{8}$ in the thin film state. (b) Polarizing optical micrographs of as-deposited and polymerized thin films deposited onto non-treated substrates. 
a

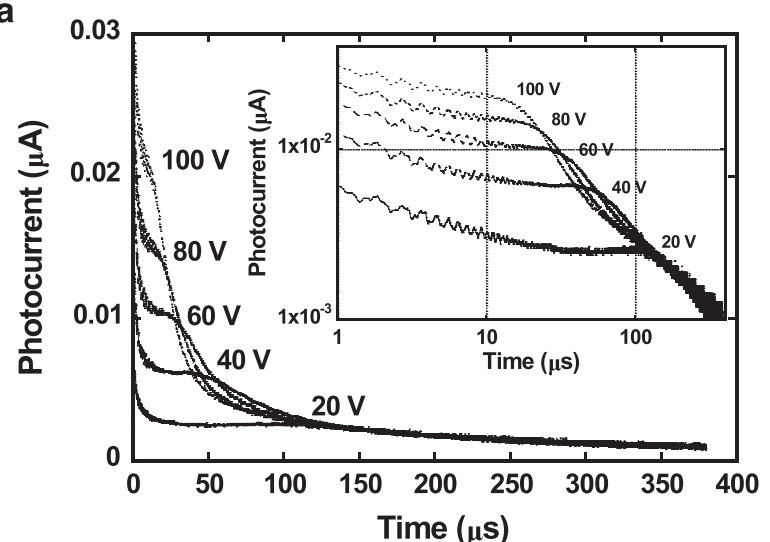

C

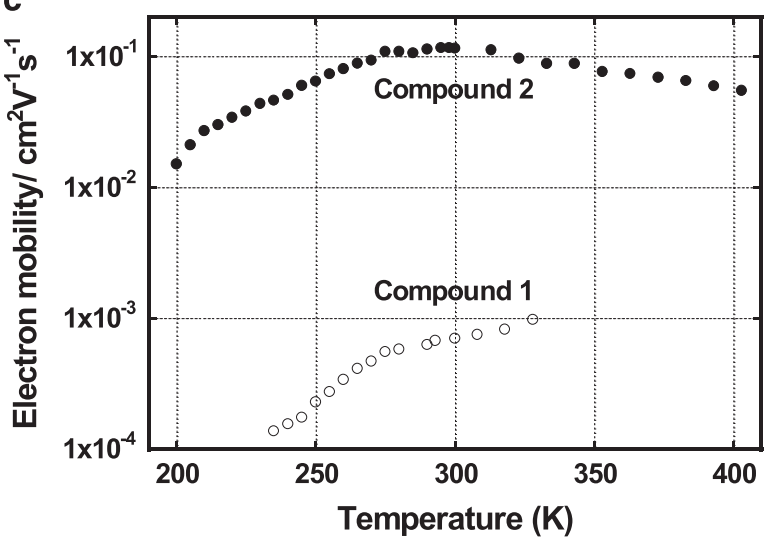

b
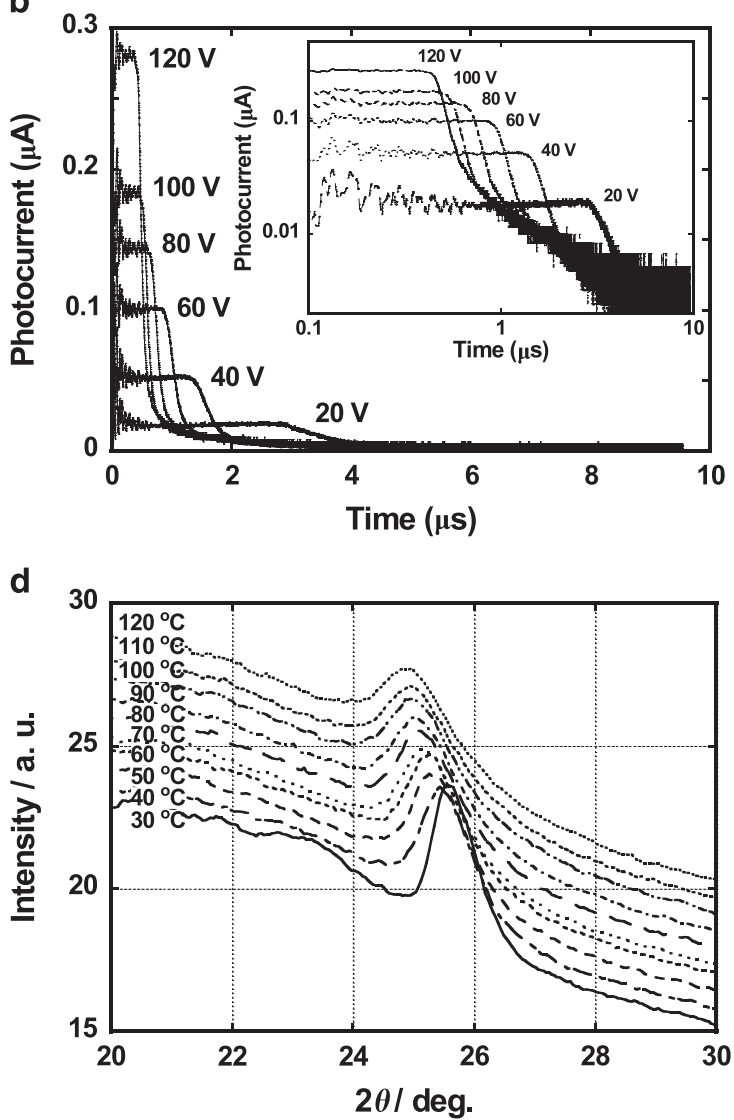

Figure 6 Transient photocurrent curves for electrons in the columnar phase of (a) compounds $\mathbf{1}$ and (b) 2 at room temperature. The sample thicknesses were $15 \mu \mathrm{m}$ for compound 1 and $25 \mu \mathrm{m}$ for compound 2. (c) Electron mobility in the columnar phase as a function of temperature. (d) X-ray diffraction patterns derived from $\pi-\pi$ stacking in the columnar aggregates of compound 2 at various temperatures.

divided into three parts. Below $0{ }^{\circ} \mathrm{C}$, the electron mobility decreases with decreasing electric field strengths and temperatures. The field dependence of the electron mobility increases with decreasing temperature. This characteristic is typical of hopping transport, which is affected by energetic disorder. The dependence on the electric field and the temperature can be described by the Gaussian disorder model, which has been used for the analysis of carrier transport characteristics in organic amorphous semiconductors. ${ }^{47}$

The field- and temperature-dependence of the electron mobility was analyzed based on a one-dimensional disorder model. ${ }^{48}$ In this model, carrier mobility $\mu$ is described by equation (1), where $T$ is the temperature, $F$ is the electric field, $\sigma$ is the energetic disorder, $\Sigma$ is the positional disorder and $C$ is a constant. The energetic disorder $\sigma$ indicates the width of the Gaussian distribution of LUMO levels of the PTCBI cores, and $\mu_{0}$ is a pre-exponential parameter corresponding to the upper limit of the electron mobility without disorder.

$$
\mu=\mu_{0} \exp \left[-\left(0.9 \frac{\sigma}{k_{\mathrm{B}} T}\right)^{2}\right] \exp \left[C\left\{\frac{\sigma}{k_{\mathrm{B}} T}-\Sigma\right\} \sqrt{F}\right]
$$

The fitting result afforded $\sigma=43 \mathrm{meV}$ and $\mu_{0}=1.2 \mathrm{~cm}^{2} \mathrm{~V}^{-1} \mathrm{~s}^{-1}$. The energetic disorder in the ordered columnar phase of compound 2 is comparable to those of the ordered smectic phases of LC terthiophene derivatives and the ordered columnar phase of a triphenylene dimer despite the presence of the bulky, thermally mobile oligosiloxane moieties. The factor $\mu_{0}$ in 2 is one order magnitude larger than $\mu_{0}$ in the ordered columnar phase of the triphenylene dimer and is comparable to the values of $\mu_{0}$ in the ordered smectic phases of terthiophene derivatives.

Above $50^{\circ} \mathrm{C}$, the electron mobility decreases with increasing temperatures. This pattern could be attributed to thermal fluctuation of the columnar aggregates. The charge carrier mobility is determined by the intermolecular $\pi$-orbital overlap. ${ }^{49}$ Figure $6 \mathrm{~d}$ displays a diffraction peak of $\sim 25^{\circ}$ in the ordered columnar phase of compound 2 at various temperatures. This peak is attributed to the $\pi-\pi$ stacking distance within the columnar aggregates. With an increase in the temperature, the diffraction angle decreases, indicating an increase in the $\pi-\pi$ stacking distance within the columnar aggregates. In this temperature range, the increase in the temperature results in the decrease of intermolecular $\pi$-orbital overlaps, which lowers the electron mobility. ${ }^{39,46}$

Between $0{ }^{\circ} \mathrm{C}$ and $50^{\circ} \mathrm{C}$, the thermal fluctuation effect should be canceled by the thermal activation process, resulting in field- and temperature-independent mobility. This result was observed in the band conduction of molecular crystals and the ordered smectic phase of phenylterthiophene. ${ }^{7}$ However, the electron transport mechanism in this ordered columnar phase is hopping transport in a disordered system, but not band-like transport because the independence of the electron mobility should be attributed to the balance between the thermal activation effect that occurs in electron hopping and the thermal fluctuation of the columnar aggregates. 
A crystal-like $\pi$-stacking structure is indispensable to the efficient transport of electronic charge carriers. In the ordered columnar phase, high electron mobility is achieved despite the presence of the oligosiloxane moieties, which are bulky compared with alkyl chains. In the ordered columnar phase, a core-shell structure in which crystal-like, one-dimensional $\pi$-stacks are surrounded by a liquid-like oligosiloxane mantle should contribute to the high electron mobility and the softness of the material. In other words, these columnar phases can be regarded as bundles of one-dimensional conductive nanowires surrounded by liquid-like, insulating cladding tubes. Compounds $\mathbf{1 - 5}$ are waxy at room temperature after melting, although red powders can be obtained by reprecipitation of compounds $\mathbf{2}$ and $\mathbf{5}$ in methanol. It is surprising that high electron mobilities that are comparable to those in molecular crystals are observed in such waxy substances.

\section{COUPLING BETWEEN ELECTRONIC FUNCTIONS AND POLAR MOIETIES IN THE NANOSEGREGATED LC PHASES}

The $\pi$-conjugated LC molecules bearing oligosiloxane moieties as well as a polar moiety also form nanosegregated LC phases in which the electronic properties can couple with the polar groups to produce new functions.

In redox-active materials, efficient ionic transport and electronic charge transport between $\pi$-conjugated chromophores are necessary. The microscopic separation between ion-conductive and electron-transporting domains should be effective for this purpose. We synthesized the PTCBI derivative 9 bearing oligosiloxane moieties as well as a triethylene oxide chain, which coordinates to metal ions, as shown in Figure 7.50 The oligosiloxane moieties do not only increase the solubilities of these compounds due to their thermal motion but also promotes the formation of soft, ordered structures due to nanosegregation.

As shown in Figure 8a, compound 9 exhibits a dimeric lamellar LC phase due to the interaction between hydrophilic triethylene oxide chains, which is in contrast to the monomeric LC phases of symmetric PTCBI derivatives bearing four oligosiloxane moieties. The lamellar LC phase is thermodynamically stable below $170{ }^{\circ} \mathrm{C}$ and does not crystallize when cooled to $-100^{\circ} \mathrm{C}$. The layer spacing determined from the $\mathrm{X}$-ray diffraction pattern is longer than the extended molecular length of compound 9 but shorter than double of that length. This result indicates that the triethylene oxide chains interdigitate. In the lamellar phase, the electron mobility, which is dependent on the electric field, is $1 \times 10^{-3} \mathrm{~cm}^{2} \mathrm{~V}^{-1} \mathrm{~s}^{-1}$ at $10^{5} \mathrm{~V} \mathrm{~cm}^{-1}$ although the carrier mobilities are field-independent in the smectic and columnar phases of conventional LC semiconductors. The strong dipole moment of the triethylene oxide chains should broaden the width of the density of states of the $\pi$ conjugated PTCBI cores, resulting in the dependence of the electron mobility on the electric field. ${ }^{50}$

Compound 9 can be mixed with lithium triflate at a concentration of $3 \mathrm{~mol} \%$. In the dimeric lamellar phase of compound $\mathbf{9}$, hydrophobic electron-conductive sublayers consisting of $\pi$-conjugated PTCBI cores and hydrophilic ion-conductive sublayers formed by polar triethylene oxide chains are laminated alternately. Lithium triflate can penetrate into the hydrophilic sublayers, interacting with the triethylene oxide chains. In the smectic phase of compound 9 containing lithium triflate, the electron mobility at room temperature decreases to $5 \times 10^{-4} \mathrm{~cm}^{2} \mathrm{~V}^{-1} \mathrm{~s}^{-1}$ at $10^{5} \mathrm{~V} \mathrm{~cm}^{-1}$. This decrease in the electron mobility suggests the presence of Coulomb interactions between electrons and ions. Compound 9 can exist as a LC mixed conductor, which can transport electrons as well as ions. ${ }^{50}$
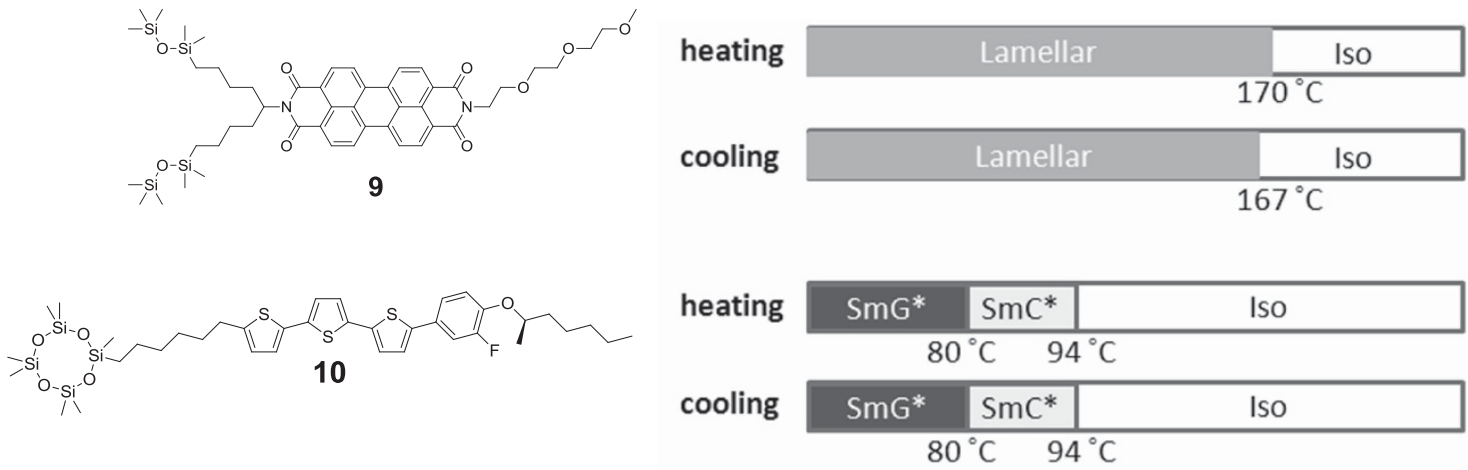

\begin{tabular}{|c|c|c|c|}
\hline heating & SmG* & SmC* & Iso \\
\hline & \multicolumn{3}{|c|}{$80^{\circ} \mathrm{C} \quad 94^{\circ} \mathrm{C}$} \\
\hline cooling & SmG* & $\mathrm{SmC}^{*}$ & Iso \\
\hline
\end{tabular}

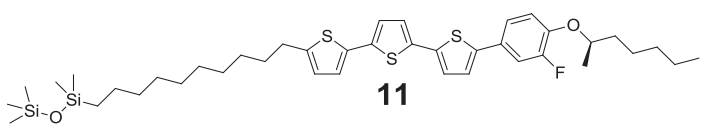

\begin{tabular}{|c|c|c|c|}
\hline heating & $\mathrm{SmF}^{*}$ & $\mathrm{SmC}^{*}$ & Iso \\
\hline & \multicolumn{3}{|c|}{$116^{\circ} \mathrm{C} 129^{\circ} \mathrm{C}$} \\
\hline cooling & $\mathrm{SmF}^{*}$ & $\mathrm{SmC}^{*}$ & Iso \\
\hline
\end{tabular}

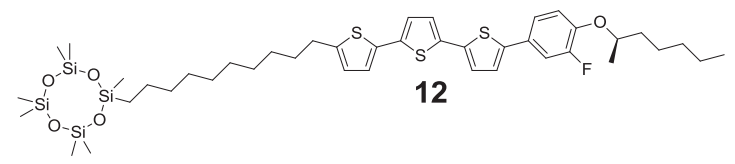

\begin{tabular}{|c|c|c|c|}
\hline heating & SmF* & SmC* & Iso \\
\hline \multicolumn{4}{|c|}{$113^{\circ} \mathrm{C} 124^{\circ} \mathrm{C}$} \\
\hline cooling & SmF* & $\mathrm{SmC}^{*}$ & Iso \\
\hline
\end{tabular}

Figure 7 Phase transition behaviors of $\pi$-conjugated liquid crystals bearing polar moieties. SmC*, chiral smectic $\mathrm{C}$ phase; SmF*, chiral smectic $\mathrm{F}$ phase; SmG*, chiral smectic G phase. 
a
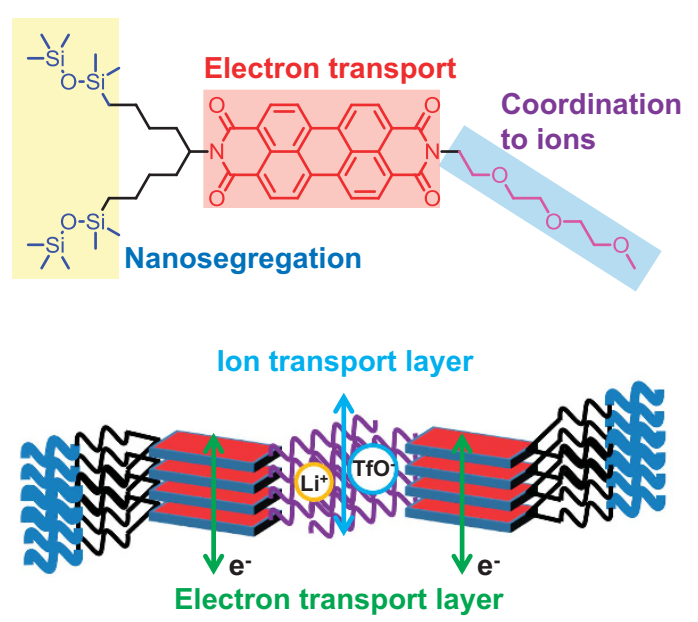

b

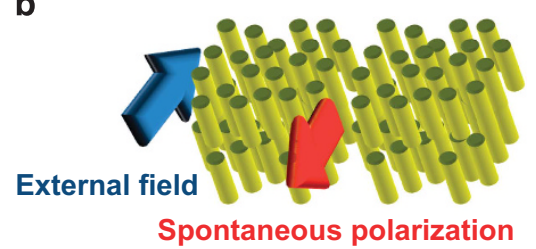

Removal of the external field

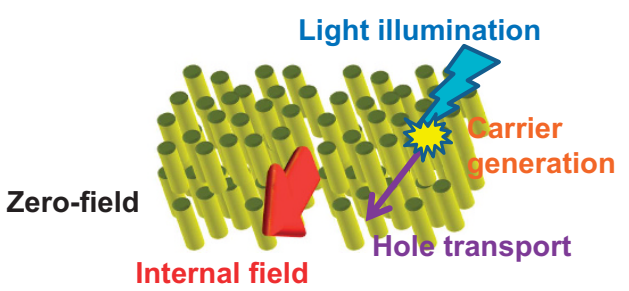

Figure 8 (a) Schematic illustration of dimeric lamellar phases of compound 9. (b) Schematic illustration of the anomalous photovoltaic effect in the $\mathrm{SmC}^{*}$ phase.

The phase transition behaviors of the fluorophenylterthiophene derivatives 10-12 bearing oligosiloxane moieties are displayed in Figure 7. These compounds exhibit a ferroelectric smectic $C^{*}$ $\left(\mathrm{SmC}^{\star}\right)$ phase. It should be noted that the fluorophenyl terthiophene derivatives bearing a bulky cyclotetrasiloxane ring also exhibit the enantiotropic $\mathrm{SmC}^{*}$ phase. ${ }^{51,52}$

In the ferroelectric $\mathrm{SmC}^{\star}$ phase of compounds 10-12, the carrier transport property derived from the lamellar aggregation of the $\pi$-conjugated phenylterthiophene units couples with the internal electric field produced by the ferroelectric spontaneous polarization, thereby causing the anomalous photovoltaic effect. ${ }^{51,52}$ In the conventional photovoltaic effect, the internal electric field is formed by the $\mathrm{p}-\mathrm{n}$ junction. Figure $8 \mathrm{~b}$ displays a schematic illustration of anomalous photovoltaic effect in the ferroelectric SmC $\mathrm{S}^{\star}$ phase. Before illumination with light, the biased application of DC produces an internal field that is retained after the removal of the external DC bias. In the anomalous photovoltaic effect, this internal electric field contributes to the dissociation of the photogenerated excitons, the transport of the holes, and the electrons to the anode and cathode. The polarity of the photovoltaic effect can be inverted by changing the polarity of the DC bias before illumination with light. Compared with the weak built-in potential based on $\mathrm{p}-\mathrm{n}$ junctions, strong internal fields can be generated in ferroelectric materials. Compounds 10-12 are able to function as ferroelectric semiconductors, which have the potential for use as new types of photovoltaic devices.

\section{CONCLUSION}

Conventional LC materials have alkyl chains that cause softness due to their thermal motion. The oligosiloxane moieties are bulky and have a tendency to form liquid-like conformations because of the low rotation potential of $\mathrm{Si}-\mathrm{O}$ bonds. However, the oligosiloxane moieties promote nanosegregation between side chains and $\pi$-conjugated cores, which is a main driving force of the appearance of the LC phases. In the columnar phases of the PTCBI derivatives bearing the oligosiloxane moieties, crystal-like $\pi$-stacks are surrounded by a liquid-like mantle consisting of the oligosiloxane moieties, which results in efficient electron transport, the appearance of LC phases at room temperature, and high solubilities in organic solvents. Even the PTCBI derivatives bearing polymerizable cyclotetrasiloxane rings exhibit LC phases at room temperature. Moreover, the thin films of the LC PTCBI derivatives with cyclotetrasiloxane rings can polymerize and be made insoluble by exposure to trifluoromethane sulfonic acid vapors. PTCBI derivatives bearing oligosiloxane moieties and a triethylene oxide chain coordinating to an ionic species exhibit a dimeric LC phase in which ion-conductive sublayers and electrontransporting $\pi$-stacks are separately self-organized. Phenylterthiophene derivatives bearing oligosiloxane moieties exhibit a ferroelectric $\mathrm{SmC}^{*}$ phase in which the anomalous photovoltaic effect is observed.

\section{CONFLICT OF INTEREST}

The authors declare no conflict of interest.

\section{ACKNOWLEDGEMENTS}

This study was financially supported by Grant-in-Aid for Scientific Research on Innovative Areas 'New Polymeric Materials Based on Element-Blocks (No. 2401)' (JSPS KAKENHI Grant Number 15H00753), Grant-in-Aid for Scientific Research (B) (JSPS KAKENHI Grant Number 15H03797) and Grant-in-Aid for Challenging Exploratory Research (JSPS KAKENHI Grant Number 15K13677), the Asahi Glass Foundation, Iketani Science and Technology Foundation, Kato Foundation for Promotion of Science, and the Tokyo Electric Power Company (TEPCO) Memorial Foundation. We thank Dr A Sonoda at the Health Research Institute of the National Institute of Advanced Industrial Science and Technology for their assistance with the NMR measurements. We also thank Professor T Ishii and Professor T Kusunose at Kagawa University for their assistance with the XRD and DSC measurements, respectively. We also thank Dr S Yazaki, Professor T Kato, Mr Y Funatsu, Miss N Takeuchi, Miss K Takenami and Mr A Seki for their collaboration in the research.

1 Sokolov, A. N., Tee, B. C.-K., Bettinger, C. J., Tok, J. B.-H. \& Bao, Z. Chemical and engineering approaches to enable organic field-effect transistors for electronic skin applications. Acc. Chem. Res. 45, 361-371 (2012).

2 Funahashi, M. Development of liquid crystalline semiconductors with high carrier mobility and their application to thin-film transistors. Polym. J. 41, 459-469 (2009).

3 Pisula, W., Zorn, M., Chang, J. Y., Müllen, K. \& Zentel, R. Liquid crystalline ordering and charge transport in semiconducting materials. Macromol. Rapid Commun. 30, 1179-1202 (2009).

4 O'Neill, M. \& Kelly, S. M. Ordered materials for organic electronics and photonics. Adv. Mater. 23, 566-584 (2011).

5 Seki, A. \& Funahashi, M. Nanostructure formation based on the functionalized side chains in liquid-crystalline heteroaromatic compounds. Heterocycles 92, 3-30 (2016).

6 Funahashi, M., Zhang, F., Tamaoki, N. \& Hanna, J. Ambipolar transport in the highly ordered smectic phase of 2-alkyl-5"-alkynylterthiophene derivative in a wide temperature range. ChemPhysChem. 9, 1465-1473 (2008). 
7 Funahashi, M., Ishii, T. \& Sonoda, A. Temperature-independent hole mobility of a smectic liquid-crystalline semiconductor based on band-like conduction. ChemPhysChem. 14, 2750-2758 (2013).

8 Aldred, M. P., Contoret, A. E. A., Farrar, S. R., Kelly, S. M., Mathieson, D., O'Neill, M., Tsoi, W. C. \& Vlachos, P. A full-color electroluminescent device and patterned photoalignment using light-emitting liquid crystals. Adv. Mater. 17, 1368-1372 (2005).

9 Funahashi, M., Zhang, F. \& Tamaoki, N. High ambipolar mobility in highly ordered smectic phase of dialkylphenylterthiophene derivative that can be applied to solution-processed organic field effect transistors. Adv. Mater. 19, 353-358 (2007).

10 Schmidt-Mende, L., Fechtenkötter, A., Müllen, K., Moons, E., Friend, R. H. \& MacKenzie, J. D. Self-organized discotic liquid crystals for high-efficiency organic photovoltaics. Science 293, 1119-1122 (2001).

11 Hori, T., Miyake, Y., Yamasaki, N., Yoshida, H., Fujii, A., Shimizu, Y. \& Ozaki, M Solution processable organic solar cell based on bulk heterojunction utilizing phthalocyanine derivative. Appl. Phys. Express 3, 101602 (2010).

12 Kitamura, T., Nakaso, S., Mizoshita, N., Tochigi, Y., Shimomura, T., Moriyama, M., Ito, K. \& Kato, T. Electroactive supramolecular self-assembled fibers comprised of doped tetrathiafulvalene-based gelators. J. Am. Chem. Soc. 127, 14769-14775 (2005).

13 Shoji, Y., Yasuda, T., Yoshio, M., Funahashi, M. \& Kato, T. Alignment of photoconductive self-assembled fibers composed of $\pi$-conjugated molecules under electric fields. J. Mater. Chem. 20, 173-179 (2010).

14 Prasanthkumar, S., Gopal, A. \& Ajayaghosh, A. Self-assembly of thienylenevinylene molecular wires to semiconducting gels with doped metallic conductivity. J. Am. Chem. Soc. 132, 13206-13207 (2010).

15 Yamamoto, Y., Fukushima, T., Suna, Y., Ishii, N., Saeki, A., Seki, S., Tagawa, S., Taniguchi, M., Kawai, T. \& Aida, T. Photoconductive coaxial nanotubes of molecularly connected electron donor and acceptor layers. Science 314, 1761-1764 (2006).

16 Takano, T., Masunaga, H., Fujiwara, A., Okuzaki, H. \& Sasaki, T. PEDOT nanocrystal in highly conductive PEDOT:PSS polymer films. Macromolecules 45, 3859-3865 (2012).

17 Kim, Y. H., Sachse, C., Machala, M. L., May, C., Müller-Meskamp, L. \& Leo, K. Highly conductive PEDOT:PSS electrode with optimized solvent and thermal post-treatment for ITO-free organic solar cells. Adv. Funct. Mater. 21, 1076-1081 (2011).

18 Brocorens, P., van Vooren, A., Chabinyc, M. L., Toney, M. F., Shkunov, M., Heeney, M. McCulloch, I., Cornil, J. \& Lazzaroni, R. Solid-state supramolecular organization of polythiophene chains containing thienothiophene units. Adv. Mater. 21 1193-1198 (2009).

19 Kato, T., Mizoshita, N. \& Kishimoto, K. Functional Liquid-crystalline assemblies: self-organized soft materials. Angew. Chem. Int. Ed. 45, 38-68 (2006).

20 Kishimoto, K., Yoshio, M., Mukai, T., Yoshizawa, M., Ohno, H. \& Kato, T. Nanostructured anisotropic ion-conductive films. J. Am. Chem. Soc. 125, 3196-3197 (2003).

21 Yoshio, M., Mukai, T., Ohno, H. \& Kato, T. One-dimensional ion transport in self-organized columnar ionic liquids. J. Am. Chem. Soc. 126, 994-995 (2004).

22 Funahashi, M. Nanostructured liquid-crystalline semiconductors - a new approach to soft matter electronics. J. Mater. Chem. C 2, 7451-7459 (2014).

$23 \mathrm{Bu}$, W., Gao, H., Tan, X., Dong, X., Cheng, X., Prehm, M. \& Tschierske, C. A bolaamphiphilic sexithiophene with liquid crystalline triangular honeycomb phase. Chem. Commun. 49, 1756-1758 (2013).

24 Yasuda, T., Ooi, H., Morita, J., Akama, Y., Minoura, K., Funahashi, M., Shimomura, T. \& Kato, T. $\pi$-Conjugated oligothiophene- based polycatenar liquid crystals: selforganization and photoconductive, luminescent, and redox properties. Adv. Funct. Mater. 19, 411-419 (2009).

25 Yazaki, S., Funahashi, M. \& Kato, T. Electrochromic liquid crystals consisting of $\pi$-conjugated and ionic moieties. J. Am. Chem. Soc. 130, 13206-13207 (2008).

26 Yazaki, S., Funahashi, M., Kagimoto, J., Ohno, H. \& Kato, T. Nanostructured liquid crystals combining ionic and electronic functions. J. Am. Chem. Soc. 132, 7702-7708 (2010).

27 Hollambya, M. J. \& Nakanishi, T. The power of branched chains: optimising functional molecular materials. J. Mater. Chem. C 1, 6178-6183 (2013).

$28 \mathrm{Lim}$, J. A., Liu, F., Ferdous, S., Muthukumar, M. \& Briseno, A. L. Polymer semiconductor crystals. Mater. Today 13, 14-24 (2010).

29 Mehl, G. H. \& Goodby, J. Liquid-crystalline, substituted octakis-(dimethylsiloxy)octasilsesquioxanes: oligomeric supermolecular materials with defined topology. Angew. Chem., Int. Ed. Engl. 35, 2641-2643 (1996).
30 Newton, J., Coles, H., Hodge, P. \& Hannington, J. Synthesis and properties of low-molar-mass liquid-crystalline siloxane derivatives. J. Mater. Chem. 4, 869-874 (1994).

31 Lagerwall, J. P. F. \& Giesselmann, F. Current topics in smectic liquid crystal research. ChemPhysChem. 7, 20-45 (2006)

32 Zelcer, A., Donnio, B., Bourgogne, C., Cukiernik, F. D. \& Guillon, D. Mesomorphism of hybrid siloxane-triphenylene star-shaped oligomers. Chem. Mater. 19, 1992-2006 (2007).

33 Matsui, A., Funahashi, M., Tsuji, T. \& Kato, T. Hole transport in liquid-crystalline polymers with a polysiloxane backbone and a phenylterthiophene moiety in the side chain. Chem. Eur. J. 16, 13465-13472 (2010).

34 Würthner, F., Saha-Möller, C. R., Fimmel, B., Ogi, S., Leowanawat, P. \& Schmidt, D. Perylene bisimide dye assemblies as archetype functional supramolecular materials. Chem. Rev. 116, 962-1052 (2015).

35 Langhals, $\mathrm{H}$. Control of the interactions in multichromophores: novel concepts. Perylene bisimides as components for larger functional units. Helv. Chim. Acta $\mathbf{8 8}$ 1309-1343 (2005).

36 Würthner, F. Perylene bisimide dyes as versatile building blocks for functional supramolecular architectures. Chem. Commun. 1564-1579 (2004).

37 Yagai, S., Usui, M., Seki, T., Murayama, H., Kikkawa, Y., Uemura, S., Karatsu, T. Kitamura, A., Asano, A. \& Seki, S. Supramolecularly engineered perylene bisimide assemblies exhibiting thermal transition from columnar to multilamellar structures. J. Am. Chem. Soc. 134, 7983-7994 (2012).

38 Funahashi, M. \& Sonoda, A. Liquid-crystalline perylene tetracarboxylic acid bisimide bearing oligosiloxane chains with high electron mobility and solubility. Org. Electron. 13, 1633-1640 (2012).

39 Funahashi, M. \& Sonoda, A. High electron mobility in a columnar phase of liquidcrystalline perylene tetracarboxylic bisimide bearing oligosiloxane chains. J. Mater. Chem. 22, 25190-25197 (2012).

40 Funahashi, M., Takeuchi, N. \& Sonoda, A. Liquid-crystalline perylene tetracarboxylic bisimide derivative bearing trisiloxan-2-yl moieties: influence on mesomorphic property and electron transport. RSC Advances 6, 18703-18710 (2016).

41 Koray, A. R., Ahsen, V. \& Bekâroğlu, O. Preparation of a novel, soluble copper phthalocyanine with crown ether moieties. J. Chem. Soc., Chem. Commun. 932-933 (1986).

42 van Nostrum, C. F., Picken, S. J., Schouten, A.-J. \& Nolte, R. J. M. Synthesis and supramolecular chemistry of novel liquid crystalline crown ether-substituted phthalocyanines: toward molecular wires and molecular ionoelectronics. J. Am. Chem. Soc. 117, 9957-9965 (1995).

43 Takagi, Y., Ohta, K., Fuji, T. \& Itoh, E. Flying-seed-like liquid crystals 2: unprecedented guidelines to obtain liquid crystalline compounds. J. Mater. Chem. 22 14418-14425 (2012)

44 Funahashi, M., Yamaoka, M., Takenami, K. \& Sonoda, A. Liquid-crystalline perylene tetracarboxylic bisimide derivatives bearing cyclotetrasiloxane moieties. J. Mater. Chem. C 1, 7872-7878 (2013)

45 Takenami, K., Uemura, S. \& Funahashi, M. In situ polymerization of liquid-crystalline thin films of electron-transporting perylene tetracarboxylic bisimide bearing cyclotetrasiloxane rings. RSC Adv. 6, 5474-5584 (2016).

46 Funahashi, M. \& Sonoda, A. Electron transport characteristics in nano-segregated columnar phases of perylene tetracarboxylic bisimide derivatives bearing oligosiloxane chains. Phys. Chem. Chem. Phys. 16, 7754-7763 (2014).

47 Hertel, D. \& Bässler, H. Photoconduction in amorphous organic solids. ChemPhysChem. 9, 666-688 (2008).

48 Bleyl, I., Erdelen, C., Schmidt, H.-W. \& Haarer, D. One-dimensional hopping transport in a columnar discotic liquid-crystalline glass. Philos. Mag. B 79, 463-475 (1999).

49 Gill, W. D. Drift mobilities in amorphous charge-transfer complexes of trinitrofluorenone and poly-n-vinylcarbazole. J. Appl. Phys. 43, 5033-5040 (1972).

50 Funahashi, M. \& Sonoda, A. Liquid-crystalline perylene tetracarboxylic bisimide derivative bearing a triethylene oxide chain and complexation of the derivative with $\mathrm{Li}$ cations. Dalton Trans. 42, 15987-15994 (2013).

51 Funatsu, Y., Sonoda, A. \& Funahashi, M. Ferroelectric liquid-crystalline semiconductors based on a phenylterthiophene skeleton: effect of introduction of oligosiloxane moieties and photovoltaic effect. J. Mater. Chem. C 3, 1982-1993 (2015).

52 Seki, A. \& Funahashi, M. Photovoltaic effects in ferroelectric liquid crystals based on phenylterthiophene derivatives. Chem. Lett. 45, 616-618 (2016).

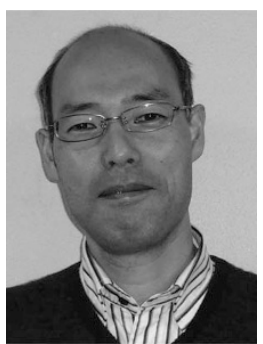

Masahiro Funahashi was born in Osaka in 1968. He graduated from the Department of Chemistry, the University of Tokyo. In 1994, he joined Prof. Jun-ichi Hanna's group of Tokyo Institute of Technology, as a research associate, and received his PhD in 1999. In 2005, he moved to the group of Dr. Nobuyuki Tamaoki in National Institute of Advanced Industrial Science and Technology (AIST), as a researcher. In 2007, he was appointed to an associate professor of the research group of Prof. Takashi Kato in the University of Tokyo. Since 2010, he has been serving as a full professor of Department of Advanced Materials Science, Kagawa University. 